\title{
Clinical Characteristics of Coronavirus Disease 2019 (COVID-19) in Patients out of Wuhan from China
}

Hua Zhang

Baoji Central Hospital

Feng Du

Xiao-jun Cao

Xia-long Feng

He-ping Zhang

Zheng-xia Wu

Bao-Feng Wang

Hong-juan Zhang

Rui Liu

Jian-jun Yang

Bo Ning

Kai Chen

ZHENPENG HUANG ( $\nabla$ huang_zhenpeng@126.com )

Cancer Center of Guangzhou Medical University https://orcid.org/0000-0003-2099-9328

\section{Research Article}

Keywords: 2019-nCoV, COVID-19, Clinical Characteristics, Wuhan, China

Posted Date: March 9th, 2020

DOI: https://doi.org/10.21203/rs.3.rs-15449/v1

License: (c) (1) This work is licensed under a Creative Commons Attribution 4.0 International License.

Read Full License 


\section{Abstract}

Background: A large-scale global outbreak of coronavirus disease-19 (COVID-19) out of Wuhan, from China, occurred in January 2020.

Objective: To examine the clinical characteristics of COVID-19in infected patients out of Wuhan, from China.

Patients and Methods: Thirteen patients were confirmed to be infected with novel coronavirus-2019 (2019-nCoV) between January 27andFebruary 8, 2020, in Baoji city, Shanxi, northwestern China. Epidemiological and clinical information, and computed to morphology imaging data from all COVID-19 patients were collected; cases were divided into two groups according to the severity of infection (mild or severe).

Results: Nine (9/13) COVID-19patientsexhibitedmild disease severity, and defined as second-generation, human-to-human transmission cases. Most patients (11/13) had a history of travel to or from Wuhan. There were no differences in sex and age between the mild and severe cases (all $P>0.05$ ). A moderate degree of fever (11/13), cough (13/13), and fatigue (8/13) were common symptoms; however, there was no statistical difference between mild and severe cases in this regard (all $P>0.05$ ). Oxyhemoglobin saturation and oxygenation index decreased, and C-reactive protein (CRP) and serum amyloid A (SAA) levels were elevated in all patients with COVID-19infection, with statistically significant differences between those with severe disease and mild infection (all $P<0.05$ ). Twelve of 13COVID-19patients exhibited changes in chest $\mathrm{CT}$ imaging features, and time course changes were different between mild and severe cases (all $P<0.05$ ).

Conclusion: Most cases of COVID-19infection were second-generation human-to-human transmissions from Wuhan and were mild in severity. The clinical characteristics of COVID-19varied.Oxyhemoglobin saturation, oxygenation index, CRP and SAA levels, and CT features were reliable parameters to evaluate the severity of COVID-19 infection. However, a few patients with mild COVID-

19diseaselackedtypicalcharacteristics such as fever and changes in CT imaging features.

\section{Introduction}

A few cases of pneumonia associated with exposure to the Wuhan Seafood market (Wuhan, China) were reported and found to be caused by novel coronavirus-2019 (2019-nCoV) in December 2019[1,2]. The 2019-nCoV infection emerged on a large scale from Wuhan and spread all over the world in January 2020,andis now known to transmitted by person-to-person contact[3].The World Health Organization (WHO) declared the outbreak of 2019-nCoVto be a public health emergency of international concern on January 30, 2020. Thenovel coronavirus was of lineage B of the genus beta-coronavirus of the coronavirus family, of which severe acute respiratory syndrome-related coronavirus (SARS-CoV) and Middle East respiratory syndrome-related coronavirus (MERS-CoV) are also included [4].The International Committee on Taxonomy of Viruses defined it as SARS-CoV-2 on February 11, 2020. The WHO also 
defined "coronavirus disease 2019" (COVID-19) in patients infected with 2019-nCoV on the same day.SARS-CoV-2is the third most fatal virus in the coronavirus family, which is weaker than MERS-CoV (37\% fatality rate) and SARS-CoV ( $10 \%$ fatality rate) [5,6].A recent study reported that fever, cough, and fatigue are common symptoms among patients infected with COVID-19[1]. However, the clinical characteristics of patients with COVID-19 remain unclear. Moreover, whether the clinical characteristics of COVID-19 are different from those of Wuhan and other regions of China has not yet been reported.

Accordingly, the present study aimed to examine the clinical characteristics of COVID-19 infection in patients out of Wuhan, Hubei, from China. We collected and analyzeddatafromCOVID-19 cases in Baoji city, Shaanxi, northwestern China.

\section{Patients And Methods}

Thirteen patients admitted to Baoji Central Hospital were confirmed to be infected with 2019nCoVusingreal-timepolymerase chain reaction (PCR) technique sat the Baoji City Center for Disease Control and Prevention (CDC) between January 27andFebruary 8, 2020.Initialonsetof disease symptoms occurred from January 18to February4, 2020.

Clinical biomedical information, including routine blood work-up, C-reactiveprotein (CRP), procalcitonin (PCT) and serum amyloid protein A (SAA), and computed tomography(CT) imaging of all COVID-19 patients with the CDC laboratory-confirmed infection with 2019-nCoV were collected at the earliest possible time.

According to the principles of diagnosis and treatment of novel coronavirus pneumonia (Revised criteria, fifth edition), COVID-19 patientswith severe respiratory distress (respiratory rate, $\geq 30$ breaths $/ \mathrm{min}$ ), hypoxemia oxyhemoglobin saturation $\leq 93 \%$, partial pressure of arterial oxygen $\left(\mathrm{PaO}_{2}\right)$ /oxygen saturation $\left(\mathrm{SpO}_{2}\right) \leq 300 \mathrm{mmHg}$, respiratory failure, shock, and multiple organ dysfunction syndrome was defined as severeCOVID-19infection [7].

The present study was approved by the Institutional Ethical Committee of Baoji Central Hospital. Verbal informed consent was obtained from all COVID-19 patients and/or their family members.

\section{Statistical Analysis}

Statistical analysis was performed using SPSS version 25.0 (IBM Corporation, Armonk, NY, USA) for Windows (Microsoft Corporation, Redmond, WA, USA). Continuous variables are expressed as mean \pm standard deviation (SD) and were compared using the Student's t test. Categorical variables are presented as proportions and percentages. Categorical variables were compared using the chi-squared test and rank sum test, respectively. Differences with $\mathrm{P}<0.05$ (two-sided) was considered to be statistically significant.

\section{Results}


A total of 13 patients with COVID-19infection ( 7 male, 6 female; sex ratio 1:1.17; mean age [ \pm SD] age, $49.54 \pm 10.85$ years [range, $27-71$ years]) were examined. Most patients were employees or farmers, and had a history of travel to or from Wuhan, or other cities in Hubei province within the preceding2 weeks. Patients out of Wuhan or Hubei province, most COVID-19 cases were second-generation human-tohuman transmissions. Disease duration ranged from 3 to 11 days, mean $6.23 \pm 2.42$ days (initial onset to confirmation of infection with 2019-nCoV). Most patients, who were from Wuhan or Hubei, were diagnosed with mild COVID-19infection (Table 1).

In those with mild COVID-19infection, there was no difference in sex; most severe COVID-19 cases were male; however, this difference was not statistically significant $(P>0.05)$ (Table 2). There were also no differences in age and disease duration between the mild and severe COVID-19 cases (all $P>0.05$ ) (Table $3)$.

\section{Characteristics of clinical symptoms in COVID-19 patients}

All COVID-19 patients exhibited symptoms of cough, and most had a moderate degree of fever; however, two did not exhibit fever. Most of those infected with COVID-19 and exhibited fever varying from 1 to 9 days (mean, $3.09 \pm 3.21$ days), and persisting 4 to 11 days (mean, $7.55 \pm 3.08$ days). Fever was not the only screening criterion for COVID-19infection. Almost one-half of COVID-19 patients exhibited expectoration, fatigue, and gastrointestinal symptoms such as anorexia, nausea, vomiting, and diarrhea. One of the COVID-19 patients experienced dyspnea and pharyngalgia. Most patients' oxyhemoglobin saturation $(90-98 \%$ [mean, $95.77 \pm 2.74 \%)$ and oxygenation index $(203-462 \mathrm{mmHg}$ [mean, $324.11 \pm 96.20$ $\mathrm{mmHg}]$ ) were decreased in the early period of2019-nCoVinfection./p>

More than one-half of patients infected byCOVID-19hadoneto three concomitant diseases, such as hypertension, diabetes, and/or cerebral infarction. One patient exhibited acute mental disorder; as such, devoting attention both physiological and mental disordersinCOVID-19 patients is important (Table 4).

\section{Characteristics of clinical symptoms and severity of COVID-19 patients}

Cough and fever were common clinical manifestations of COVID-19. There were no differences between mild and severe cases; however, a few patients with mild COVID-19disease did not exhibit fever. Severe COVID-19 cases were more likely to experience expectoration and fatigue; however, the difference was not statistically significant (all $P>0.05$ ). Oxyhemoglobin saturation and oxygenation were both sensitive indices for identification of mild and severe COVID-19 (all $P<0.05$ ). Patients with severe COVID-19 were significantly more likely to exhibit hypoxia and respiratory distress (Table 5).

The present study also confirmed that patients with concomitant diseases were more likely to deteriorate in the early stagesofCOVID-19infection $(P<0.05)$ (Table 6). 
In the early period of COVID-19 infection, routine blood work-up, including white blood cells, neutrophils, lymphocytes and monocytes, did not change significantly, and white blood cells and neutrophils were higher in severe cases than in mild cases (all $P<0.05$ ).

PCT levels did not increase in the early period of COVID-19infection, and there was no difference between those with mild and severe disease. In contrast, CRP and SAA levels increased rapidly in all COVID-19 cases, and the levels of these two biomarkers were significantly higher in those with severe disease than in mild cases (all $P<0.05$ ). As such, CRP and SAA may be sensitive indices for detecting COVID-19 and classifying disease severity (Table 7).

\section{CT imaging changes in COVID-19 patients}

Almost every COVID-19 patient exhibited imaging changes on chest CT scans, with bilateral lung lesions in severe cases, with both unilateral and bilateral lung lesions in patients with mild infection. However, one patient with mild disease did not exhibit significant lung lesions $(u-=3.1542, P<0.05)$.

Chest CT scan image changes, such as multiple patchy sub-segmental or segmental ground-glass opacities, shadows or consolidations in the bilateral lung, were found among COVID-19 patients. Five days after confirmation of infection with 2019-nCoV, CT imaging revealed that shadows or consolidations were absorbed in the lungs in more than one-half of mild cases; however, this was exacerbated in most severe cases $(u-=1.9748, P<0.05)$. Chest CT scan was an effective method to examine and evaluate the severity of COVID-19infection (Table 8,Figure 1).

\section{Discussion}

In the present study, 13 individuals were confirmed to be infected with 2019-nCoV using real-time PCR techniques. Studies have shown that real-time PCR is one of the most accurate and effective methods to detect 2019-nCoV infection[3,8]. Most patients with COVID-19 (11 of 13 cases) in the present study either traveled to Wuhan, Hubei, or were exposed to an individual who returned from Hubei in early January 2020.Two patients had no history of travel or contract during the time period. Most COVID-19 patients out of Wuhan or Hubei were cases involving second-generation human-to-human transmission; the duration from initial onset to confirm infected 2019-nCoV was 3-11 days (mean, 6.23 \pm 2.42 days), and there were no statistical differences between mild and severe cases. There was potentially large firstgeneration human-to-human 2019-nCoV transmission in Wuhan, and Wuhan was also the major hub for the spread of the 2019-nCoVoutbreak in other cities for approximately1-2 weeks[9-12].

Results of our study suggest that there are no differences between age and sex with 2019-nCoV infection and the severity of COVID-19. However, patients with concomitant diseases, such as hypertension, diabetes and/or cerebral infarction, were significantly more likely to develop severe COVID-19-related disease. Another study suggested that elderly individuals experience faster disease progression and death[13]. 
A moderate degree of fever, cough, fatigue, and gastrointestinal symptoms, such as anorexia, nausea, vomiting and diarrhea, were common symptoms among COVID-19 patients. A few patients experienced expectoration, dyspnea, and pharyngalgia, among others, which was similar to previous reports $[3,4]$. However, in our study, two patients with mild COVID-19 infection did not exhibit fever, suggesting that fever was not the only clinical characteristic of COVID-19, which was different from COVID-19 patients in Wuhan[14,15]. There is a difference between mild and severe cases in terms of degree of fever, days of persistent fever, cough, expectoration, fatigue, and other symptoms. Oxyhemoglobin saturation and oxygenation index are sensitive markers to evaluate the severity ofCOVID-19. Oxyhemoglobin saturation and oxygenation index decreased in the early period in cases with severeCOVID-19; in contrast, most mild cases were normal[16].

CRP and SAA levels maybe effective biomedical indices for detecting COVID-19 infection. CRP and SAA levels increased rapidly during the early period of 2019-nCoV infection; moreover, indices of severe cases increased to higher levels than those with mild disease. However, white blood cells, neutrophils, lymphocytes, and monocytes demonstrated no significant changes during the early period of infection in both mild and severe cases. PCT levels also did not change.

Most COVID-19 patients in our study exhibited significant changes in chest CT imaging features. Chest CT is very important for the initial diagnosis of COVID-19[17]. Chest CT may be helpful in diagnosing individuals with high clinical suspicion of 2019-nCoV infection but negative RT-PCR screening[18].Typical CT imaging features include ground-glass densities, shadows or consolidations in both lungs[19,20].A recent study reported that in patients with mild COVID-19 infection, lung abnormalities on chest CT scan would recover approximately 10 days after the initial onset of symptoms[21]. In our study, most patients with mild COVID-19 $(n=5)$ chest CT abnormalities were gradually absorbed 5 days after confirmation of infection; however, most patients with severe disease experienced exacerbation of symptoms.

However, one mild COVID-19 case showed no significant chest CT image changes, which differed from Wuhan COVID-19 patients[22].

In conclusion, results of the present study confirmed that most COVID-19 infections were secondgeneration human-to-human transmissions from other cities in China out of Wuhan, Hubei. The typical clinical characteristics of COVID-19 include a moderate degree of fever, cough, fatigue, and gastrointestinal symptoms. Oxyhemoglobin saturation and oxygenation index changed significantly in severe cases. Serum levels of CRP and SAA were reliable biomarkers to evaluate the severity of COVID19 infection. Chest CT was another effective method of detecting infection with2019-nCoV. However, a few patients with mild COVID-19 disease without fever and CT imaging changes require the combination of additional screening methods.

\section{References}

[1]Chaolin Huang, Yeming Wang, Xingwang Li, Lili Ren, Jianping Zhao, Yi Hu, Li Zhang, Guohui Fan, Jiuyang Xu, Xiaoying Gu, Zhenshun Cheng, Ting Yu, Jiaan Xia, Yuan Wei, Wenjuan Wu, Xuelei Xie, Wen 
Yin, Hui Li, Min Liu, Yan Xiao, Hong Gao, Li Guo, Jungang Xie, Guangfa Wang, Rongmeng Jiang, Zhancheng Gao, Qi Jin, Jianwei Wang, Bin Cao. Clinical features of patients infected with 2019 novel coronavirus in Wuhan, China. Lancet, 2020 Jan 24. [Online ahead of print] https://doi.org/10.1016/S0140-6736(20)30183-5

[2]Qun Li, Xuhua Guan, Peng Wu, Xiaoye Wang, Lei Zhou, Yeqing Tong, Ruiqi Ren, Kathy S M Leung, Eric H Y Lau, Jessica Y Wong, Xuesen Xing, Nijuan Xiang, Yang Wu, Chao Li, Qi Chen, Dan Li, Tian Liu, Jing Zhao, Man Li, Wenxiao Tu, Chuding Chen, Lianmei Jin, Rui Yang, Qi Wang, Suhua Zhou, Rui Wang, Hui Liu, Yingbo Luo, Yuan Liu, Ge Shao, Huan Li, Zhongfa Tao, Yang Yang, Zhiqiang Deng, Boxi Liu, Zhitao Ma, Yanping Zhang, Guoqing Shi, Tommy T Y Lam, Joseph T K Wu, George F Gao, Benjamin J Cowling, Bo Yang, Gabriel M Leung, Zijian Feng. Early transmission dynamics in Wuhan, China, of novel coronavirus-infected pneumonia. N Engl J Med, 2020 Jan 29 [Online ahead of print]. https://doi.org/10.1056/NEJMoa2001316.

[3]Yingxia Liu, Yang Yang, Cong Zhang, Fengming Huang, Fuxiang Wang, Jing Yuan, Zhaoqin Wang, Jinxiu Li, Jianming Li, Cheng Feng, Zheng Zhang, Lifei Wang, Ling Peng, Li Chen, Yuhao Qin, Dandan Zhao, Shuguang Tan, Lu Yin, Jun Xu, Congzhao Zhou , Chengyu Jiang, Lei Liu. Clinical and biochemical indexes from 2019-nCoV infected patients linked to viral loads and lung injury. Sci China Life Sci, 2020 Feb 9 [Online ahead of print].

https://doi.org/10.1007/s11427-020-1643-8

[4]Na Zhu, Dingyu Zhang, Wenling Wang, Xinwang Li, Bo Yang, Jingdong Song, Xiang Zhao, Baoying Huang, Weifeng Shi, Roujian Lu, Peihua Niu, Faxian Zhan, Xuejun Ma, Dayan Wang, Wenbo Xu, Guizhen Wu, George F Gao, Wenjie Tan, China Novel Coronavirus Investigating and Research Team. A novel coronavirus from patients with pneumonia in China, 2019. N Engl J Med, 2020 Jan 24 [Online ahead of print].

doi: 10.1056/NEJMoa2001017.

[5]Yong Jiang, Jun Xu, Chengzhi Zhou, Zhenguo Wu, Shuqing Zhong, Jinghua Liu, Wei Luo, Tao Chen, Qinghe Qin, Peng Deng. Characterization of cytokine/ chemokine profiles of severe acute respiratory syndrome. Am J Respir Crit Care Med. 2005; 171(8):850-857.

[6]Peihua Niu, Guangyu Zhao, Yao Deng, Shihui Sun, Wenling Wang, Yusen Zhou, Wenjie Tan. A novel human mAb (MERS-GD27) provides prophylactic and postexposure efficacy in MERS-CoV susceptible mice. Sci China Life Sci. 2018;61(10):1280-1282.

[7]National Health Commssion of the People's Republic of China, 2020. Precept on diagnosis and treatment of novel coronavirus pneeumoia (The fifth version; Revision version). Available from: <http://www.nhc.gov.cn/yzygj/s7653p/202002/d4b895337e19445f8d728fcaf1e3e13a.shtml>. [8]Victor M Corman, Olfert Landt, Marco Kaiser, Richard Molenkamp, Adam Meijer, Daniel Kw Chu, Tobias Bleicker, Sebastian Brünink, Julia Schneider, Marie Luisa Schmidt, Daphne Gjc Mulders, Bart L Haagmans, Bas van der Veer, Sharon van den Brink, Lisa Wijsman, Gabriel Goderski, Jean-Louis Romette, Joanna Ellis, Maria Zambon, Malik Peiris, Herman Goossens, Chantal Reusken, Marion Pg Koopmans, Christian Drosten. Detection of 2019 novel coronavirus (2019-nCoV) by real-time RT-PCR. Euro Surveill, 2020; 25 (3):1-8.

[9]Xingguang LI, Junjie ZAl, Xiaomei Wang, Yi Li. Potential of large 'first generation' human-to-human 
transmission of 2019-nCoV. J Med Virol, 2020 Jan 30 [Online ahead of print].

doi: $10.1002 / j m v .25693$

[10]Joseph T Wu, Kathy Leung, Gabriel M Leung. Nowcasting and forecasting the potential domestic and international spread of the 2019-nCoV outbreak originating in Wuhan, China: a modelling study. Lancet. 2020 Jan 31 [Online ahead of print].

doi: 10.1016/S0140-6736(20)30260-9

[11]Shi Zhao, Qianyin Lin, Jinjun Ran, Salihu S Musa, Guangpu Yang, Weiming Wang, Yijun Lou, Daozhou Gao, Lin Yang, Daihai He, Maggie H Wang. Preliminary estimation of the basic reproduction number of novel coronavirus (2019-nCoV) in China, from 2019 to 2020: A data-driven analysis in the early phase of the outbreak. Int J Infect Dis, 2020 Jan 30 [Online ahead of print]

doi: 10.1016/j.ijid.2020.01.050

[12]Tao Zhou, Quanhui Liu, Zimo Yang, Jingyi Liao, Kexin Yang, Wei Bai, Xin Lu, Wei Zhang. Preliminary prediction of the basic reproduction number of the Wuhan novel coronavirus 2019-nCoV. J Evid Based Med, 2020 Feb 12 [Online ahead of print]

doi: $10.1111 / \mathrm{jebm} .12376$

[13]Weier Wang, Jianming Tang, Fangqiang Wei. Updated understanding of the outbreak of 2019 novel coronavirus (2019-nCoV) in Wuhan, China. J Med Virol. 2020 Jan 29 [Online ahead of print].

doi: $10.1002 / j m v .25689$

[14]Chaolin Huang, Yeming Wang, Xingwang Li, Lili Ren, Jianping Zhao, Yi Hu, Li Zhang, Guohui Fan, Jiuyang Xu, Xiaoying Gu, Zhenshun Cheng, Ting Yu, Jiaan Xia, Yuan Wei, Wenjuan Wu, Xuelei Xie, Wen Yin, Hui Li, Min Liu, Yan Xiao, Hong Gao, Li Guo, Jungang Xie, Guangfa Wang, Rongmeng Jiang, Zhancheng Gao, Qi Jin, Jianwei Wang, Bin Cao. Clinical features of patients infected with 2019 novel coronavirus in Wuhan, China. Lancet, 2020 Jan 24 [Online ahead of print].

doi: 10.1016/S0140-6736(20)30183-5

[15]Nanshan Chen, Min Zhou, Xuan Dong, Jieming Qu, Fengyun Gong, Yang Han, Yang Qiu, Jingli Wang, Ying Liu, Yuan Wei, Jia'an Xia, Ting Yu, Xinxin Zhang, Li Zhang. Epidemiological and clinical

characteristics of 99 cases of 2019 novel coronavirus pneumonia in Wuhan, China: a descriptive study. Lancet, 2020 Jan 30 [Online ahead of print].

doi: 10.1016/S0140-6736(20)30211-7

[16]Ying-Hui Jin, Lin Cai, Zhen-Shun Cheng, Hong Cheng, Tong Deng, Yi-Pin Fan, Cheng Fang, Di Huang, Lu-Qi Huang, Qiao Huang, Yong Han, Bo Hu, Fen Hu, Bing-Hui Li, Yi-Rong Li, Ke Liang, Li-Kai Lin, Li-Sha Luo, Jing Ma, Lin-Lu Ma, Zhi-Yong Peng, Yun-Bao Pan, Zhen-Yu Pan, Xue-Qun Ren, Hui-Min Sun, Ying Wang, Yun-Yun Wang, Hong Weng, Chao-Jie Wei, Dong-Fang Wu, Jian Xia, Yong Xiong, Hai-Bo Xu, XiaoMei Yao, Yu-Feng Yuan, Tai-Sheng Ye, Xiao-Chun Zhang, Ying-Wen Zhang, Yin-Gao Zhang, Hua-Min Zhang, Yan Zhao, Ming-Juan Zhao, Hao Zi, Xian-Tao Zeng, Yong-Yan Wang, Xing-Huan Wang, for the Zhongnan Hospital of Wuhan University Novel Coronavirus Management and Research Team, EvidenceBased Medicine Chapter of China International Exchange and Promotive Association for Medical and Health Care (CPAM). A rapid advice guideline for the diagnosis and treatment of 2019 novel coronavirus (2019-nCoV) infected pneumonia (standard version). Mil Med Res. 2020; 7(1):4.

[17]Yueying Pan, Hanxiong Guan. Imaging changes in patients with 2019-nCov. Eur Radiol. 2020 Feb 6 
[published online ahead of print].

doi:10.1007/s00330-020-06713-z

[18]Xingzhi Xie, Zheng Zhong, Wei Zhao, Chao Zheng, Fei Wang, Jun Liu. Chest CT for Typical 2019-nCoV Pneumonia: Relationship to Negative RT-PCR Testing. Radiology. 2020 Feb 12 [Online ahead of print]. doi: $10.1148 /$ radiol.2020200343

[19]Ya-ni Duan, Jie Qin. Pre- and Posttreatment Chest CT Findings: 2019 Novel Coronavirus (2019-nCoV) Pneumonia. Radiology. 2020 Feb 12 [Online ahead of print].

doi: $10.1148 /$ radiol.2020200323

[20]Michael Chung, Adam Bernheim, Xueyan Mei, Ning Zhang, Mingqian Huang, Xianjun Zeng, Jiufa Cui, Wenjian Xu, Yang Yang, Zahi A Fayad, Adam Jacobi, Kunwei Li, Shaolin Li, Hong Shan. CT Imaging Features of 2019 Novel Coronavirus (2019-nCoV). Radiology, 2020 Feb 4 [Online ahead of print]. doi: 10.1148/radiol.2020200230

[21]Xingzhi Xie, Zheng Zhong,Wei Zhao, Chao Zheng, Fei Wang, Jun Liu. Chest CT for Typical 2019-nCoV Pneumonia: Relationship to Negative RT-PCR Testing. Radiology. 2020 Feb 12 [Online ahead of print]. doi: 10.1148/radiol.2020200343

[22]Yueying Pan, Hanxiong Guan, Shuchang Zhou, Yujin Wang, Qian Li, Tingting Zhu, Qiongjie Hu, Liming Xia. Initial CT findings and temporal changes in patients with the novel coronavirus pneumonia (2019nCoV): a study of 63 patients in Wuhan, China. Eur Radiol, 2020 Feb 13 [Online ahead of print]. doi: 10.1007/s00330-020-06731-x

\section{Tables}

Table 1 Epidemiological Characteristics of COVID-19 Patients 


\begin{tabular}{|lc|}
\hline \multicolumn{2}{|l|}{ Epidemiological Characteristics } \\
\hline Gender & $7(53.8 \%)$ \\
\hline Male & $6(46.2 \%)$ \\
\hline Female & $27-71(49.54 \pm 10.85)$ \\
\hline Age (Years) & \\
\hline Occupation & $5(46.2 \%)$ \\
\hline Employee & $5(38.4 \%)$ \\
\hline Farmer & $1(7.7 \%)$ \\
\hline Teacher & $1(7.7 \%)$ \\
\hline Unemployed & $11(84.62 \%)$ \\
\hline History of Travel to or From Wuhan, Hubei & $2(15.38 \%)$ \\
\hline Yes & \\
\hline No & \\
\hline Patients' Generation of Human-to-human Transmission & \\
\hline First Generation & $2(15.38 \%)$ \\
\hline Second Generation & $9(69.24 \%)$ \\
\hline Third Generation & $2(15.38 \%)$ \\
\hline From Initial Onset to Confirm Infected 2019-nCoV (Day) & \\
\hline $3-11$ (6.23 \pm 2.42$)$ & \\
\hline Severity of CoVID-19 & \\
\hline Mild & \\
\hline Severe & \\
\hline & \\
\hline
\end{tabular}

Table 2 Gender Characteristics of Different Severity of COVID-19

\begin{tabular}{|lll|}
\hline Gender \# & Male & Female \\
\hline Mild Cases $(n=9)$ & $4(44.44 \%)$ & $5(55.56 \%)$ \\
\hline Severe Cases $(n=4)$ & $3(75 \%)$ & $1(25 \%)$ \\
\hline
\end{tabular}


$\# x^{2}=0.1741, P>0.05$

Table 3 Ages and Diagnosis Time in Different Severity of COVID-19 Cases

\begin{tabular}{|ll|}
\hline \multicolumn{2}{|l|}{ Ages and Diagnosis Time } \\
\hline Age (Year) & $27-71(47.00 \pm 11.74)$ \\
\hline Mild Cases & $50-64(55.25 \pm 6.40)$ \\
\hline Severe Cases & $t=-1.301, P>0.05$ \\
\hline & $3-11(6.67 \pm 2.69)$ \\
\hline From Initial Onset to Confirm Infected $2019-n C o V$ (Day)
\end{tabular}

Table 4 Clinical Symptoms and Concomitant Diseases in COVID-19 Patients 


\begin{tabular}{|ll|}
\hline Clinical Symptoms and Concomitant Diseases \\
\hline Fever & $11(84.62 \%)$ \\
\hline Yes & $2(15.38 \%)$ \\
\hline No & $1-9(3.09 \pm 3.21)$ \\
\hline From Initial Onset to to Fever (Day) & $38.0-39.0(38.35 \pm 0.40)$ \\
\hline Degree of Fever $\left({ }^{\circ} \mathrm{C}\right)$ & $4-11(7.55 \pm 3.08)$ \\
\hline Day of Persistent Fever & $13(100 \%)$ \\
\hline Cough & $5(38.46 \%)$ \\
\hline Expectoration & $8(61.54 \%)$ \\
\hline Fatigue & $6(46.15 \%)$ \\
\hline Gastrointestinal Symptoms & $1(7.69 \%)$ \\
\hline Dyspnea & $1(7.69 \%)$ \\
\hline Pharyngalgia & $90-98(95.77 \pm 2.74)$ \\
\hline Oxyhemoglobin Saturation $(\%)$ & $203-462(324.11 \pm 96.20)$ \\
\hline Oxygenation Index (mmHg) & $3(37.5 \%)$ \\
\hline Concomitant Diseases & $3(37.5 \%)$ \\
\hline Yes & $2(25 \%)$ \\
\hline No & $5(38.46 \%)$ \\
\hline Numbers of Concomitant Diseases & \\
\hline 1 & \\
\hline 2 & \\
\hline 3 & \\
\hline
\end{tabular}

Table 5 Clinical Symptoms in Different Severity of COVID-19 Cases 


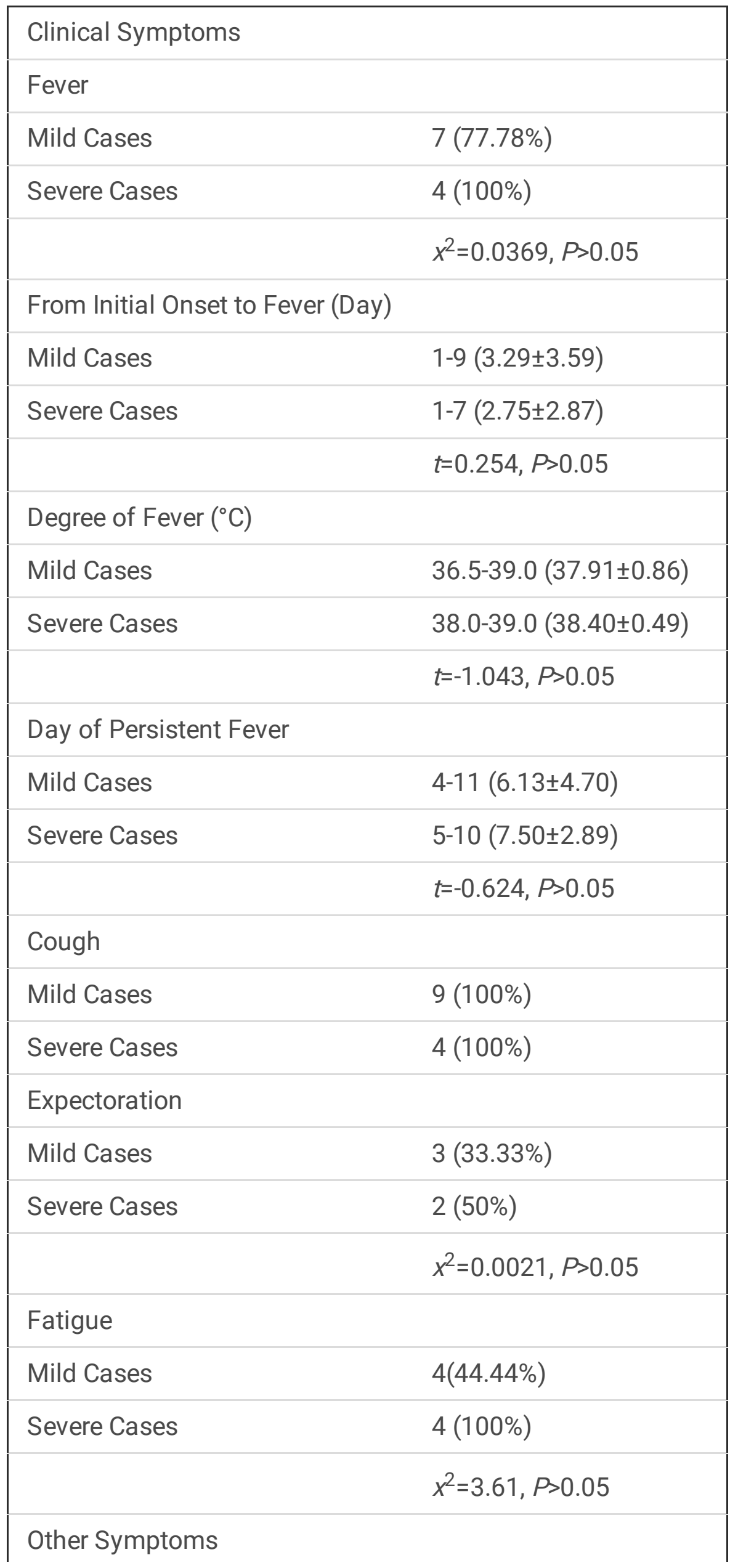




\begin{tabular}{|ll|}
\hline Mild Cases & $5(55.56 \%)$ \\
\hline Severe Cases & $3(75 \%)$ \\
\hline Oxyhemoglobin Saturation (\%) & $x^{2}=0.4424, P>0.05$ \\
\hline Mild Cases & $95-98(97.00 \pm 1.80)$ \\
\hline Severe Cases & $90-94(93.00 \pm 2.58)$ \\
\hline & $t=3.255, P<0.05$ \\
\hline Oxygenation Index $(\mathrm{mmHg})$ & \\
\hline Mild Cases & $342-462(398.60 \pm 50.91)$ \\
\hline Severe Cases & $203-252(231.00 \pm 20.41)$ \\
\hline & $t=6.133, P<0.05$ \\
\hline
\end{tabular}

Table 6 Concomitant Diseases in Different Severity of COVID-19 Cases

\begin{tabular}{|lllll|}
\hline Numbers of Concomitant Diseases & 0 & 1 & 2 & 3 \\
\hline Mild COVID-19 & $5(55.56 \%)$ & $3(33.33 \%)$ & $1(11.11 \%)$ & 0 \\
$(\mathrm{n}=9)$ & & & & \\
\hline Severe COVID-19 $(\mathrm{n}=4)$ & 0 & 0 & 2 & 2 \\
& & & $(50 \%)$ & $(50 \%)$ \\
\hline
\end{tabular}

$\# x^{2}=11.0963, P<0.05$

Table 7 Blood Biomedical Characteristics of COVID-19 Patients 


\begin{tabular}{|lllll|}
\hline & Total Cases & Mild Cases $^{*}$ & Severe Cases & \\
\hline White Blood Cell $\left(\times 10^{9} / \mathrm{L}\right)$ & $3.97 \pm 0.89$ & $3.58 \pm 0.70$ & $4.85 \pm 0.60$ & $* t=-3.132, P<0.05$ \\
\hline Neutrophil $\left(\times 10^{9} / \mathrm{L}\right)$ & $2.46 \pm 0.76$ & $2.12 \pm 0.56$ & $3.25 \pm 0.52$ & $* t=-3.410, P<0.05$ \\
\hline Lymphocyte $\left(\times 10^{9} / \mathrm{L}\right)$ & $0.98 \pm 0.21$ & $0.99 \pm 0.25$ & $0.95 \pm 0.09$ & $* t=0.368, P>0.05$ \\
\hline Monocyte $\left(\times 10^{9} / \mathrm{L}\right)$ & $0.48 \pm 0.25$ & $0.41 \pm 0.18$ & $0.63 \pm 0.34$ & $* t=-1.586, P>0.05$ \\
\hline CRP $(\mathrm{mg} / \mathrm{L})$ & $20.07 \pm 24.66$ & $9.84 \pm 11.90$ & $43.09 \pm 32.17$ & $* t=-2.819, P<0.05$ \\
\hline PCT $(\mathrm{ng} / \mathrm{L})$ & $0.04 \pm 0.03$ & $0.03 \pm 0.02$ & $0.05 \pm 0.03$ & $* t=-0.11, P>0.05$ \\
\hline SAA $(\mathrm{mg} / \mathrm{L})$ & $93.00 \pm 111.17$ & $36.41 \pm 47.18$ & $268.93 \pm 33.13$ & $* t=-8.835, P<0.05$ \\
\hline
\end{tabular}

Table 8 CT Imaging Changes in COVID-19 Patients

\begin{tabular}{|c|c|c|}
\hline & Mild Cases $(n=9)$ & Severe Cases $(n=4)$ \\
\hline \multicolumn{3}{|l|}{ Location of Lung lesion } \\
\hline No Lung Lesion & $1(11.11 \%)$ & 0 \\
\hline Unilateral Lung Lesion & $1(11.11 \%)$ & 0 \\
\hline Bilateral Lung Lesion & $7(77.78 \%)$ & $4(100 \%)$ \\
\hline \multicolumn{3}{|c|}{$u=3.1542, P<0.05$} \\
\hline \multicolumn{3}{|c|}{ Day 5 After Confirmed Infection of 2019-nCoV CT Imaging Change } \\
\hline Exacerbation & $2(22.22 \%)$ & $3(75 \%)$ \\
\hline No change & $2(22.22 \%)$ & 0 \\
\hline \multirow[t]{2}{*}{ Absorption } & $5(55.56 \%)$ & $1(25 \%)$ \\
\hline & $u=1.9748, P<0.05$ & \\
\hline
\end{tabular}

\section{Figures}



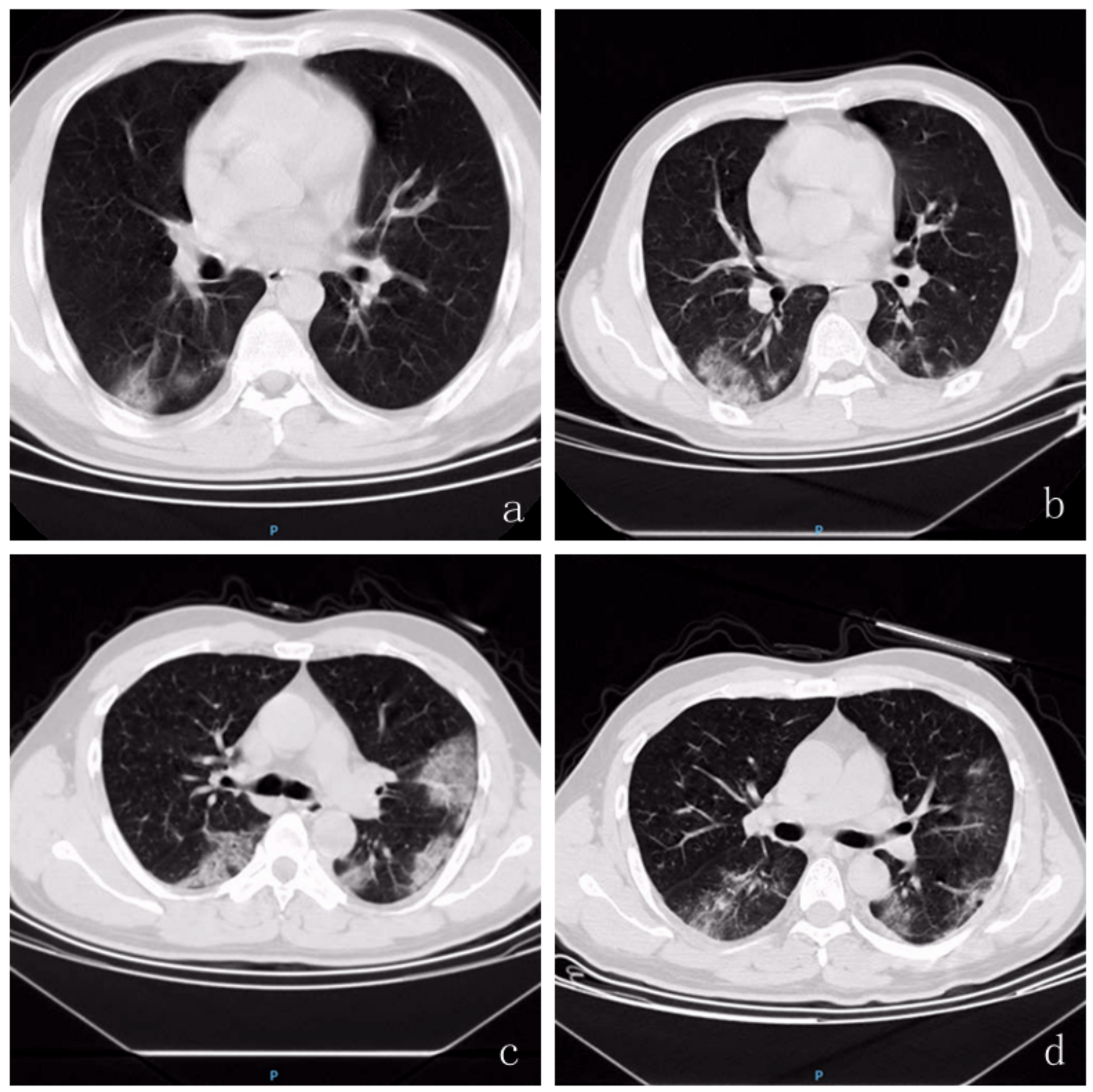

\section{Figure 1}

At the day of confirm infection of 2019-nCoV, chest CT showed that multiple patchy sub-segmental or segmental ground-glass density shadow and consolidation were in bilateral lung (a. severe cases \& $\mathrm{C}$. mild cases); b. Severe COVID-19 patient 5 days after confirm infection of 2019-nCoV, ground-glass density shadow and consolidation in bilateral lung was exacerbation; d. Mild COVID-19 patient 5 days after confirm infection of 2019-nCoV, ground-glass density shadows in bilateral lung was absorbed. 